\title{
Characteristics of Sleep in a Pediatric Population Seeking Mental Health Services
}

\author{
Kimberly E. Hunter, Michele S. Knox \\ Department of Psychiatry, University of Toledo College of Medicine and Life Sciences, Toledo, USA \\ Email: kimberly.hunter@utoledo.edu
}

How to cite this paper: Hunter, K. E., \& Knox, M. S. (2017). Characteristics of Sleep in a Pediatric Population Seeking Mental Health Services. Psychology, 8, 2534-2546. https://doi.org/10.4236/psych.2017.814160

Received: October 26, 2017

Accepted: December 25, 2017

Published: December 27, 2017

Copyright (C) 2017 by authors and Scientific Research Publishing Inc. This work is licensed under the Creative Commons Attribution International License (CC BY 4.0).

http://creativecommons.org/licenses/by/4.0/

\begin{abstract}
This study explored characteristics of sleep and other presenting problems in children and adolescents seeking mental health services within an outpatient clinic. Primary caregivers seeking outpatient mental health services for their children between the ages of $2-18$ completed a measure assessing various aspects of their children's sleep, emotional and behavioral problems, and use of electronics at bedtime. Results showed reduced sleep durations compared to recommended guidelines. Results also indicated that sleep duration was negatively associated with the total number of problems listed for both the youngest and school aged groups. Children and adolescents with worries and fears, irritability, and mood swings had significantly less sleep, and participants with sadness had longer sleep latencies. Regarding use of electronics, participants with televisions in the bedroom reported significantly less hours of sleep than those without. The average number of hours of television watched per week was positively correlated with the number of minutes of sleep latency, while videogame usage was negatively correlated with sleep duration. The study results indicated that sleep disturbances are significant issues impacting youth who are experiencing mental health issues. These results also emphasize the importance of a thorough sleep assessment for children who present for mental health services.
\end{abstract}

\section{Keywords}

Sleep Problems, Children, Adolescents, Mental Health, Media Use

\section{Introduction}

Sleep disturbances are common problems in childhood and adolescence. $\mathrm{Nu}$ merous studies have found that anywhere between $25 \%$ - 40\% of children and adolescents world-wide experience some types of sleep disturbance (Mindell, 
Kuhn, Lewin, Meltzer, \& Sadeh, 2006; Owens, 2008). Sleep disturbances may include difficulty settling, sleep onset delay/insomnia, night wakings, excessive daytime fatigue, nightmares, parasomnias, bedtime refusal, and sleep disordered breathing problems such as obstructive sleep apnea. Two of the most common sleep problems are night wakings and difficulty settling and falling asleep at bedtime (Waters, Suresh, \& Nixon, 2013; Lavigne et al., 1999). Common causes of sleep disturbances include a variety of medical, psychological, developmental, and environmental factors which in isolation or in combination may contribute to or maintain a child's sleep disturbances.

Sleep needs change over time, and total sleep time needed tends to decline as the individual ages. According to the National Heart, Lung, and Blood Institute, newborns require 16 - 18 hours per day, preschool aged children require 11 - 12 hours per day, school aged children require at least 10 hours per day, and adolescents require 9 - 10 hours per day (National Heart, Lung, and Blood Institute, 2017). However, many children do not receive adequate sleep due to various sleep disturbances, leading to daytime sleepiness as well as a multitude of other negative outcomes (Fallone, Owens, \& Deane, 2002). The negative consequences of sleep problems in children and adolescents have been well documented and include mood disturbances, anxiety, behavior problems, neurocognitive deficits, hyperactivity, and academic and learning struggles (Fallone et al., 2002; Mindell et al., 2006; Owens, 2008). Additionally, research has found that delayed sleep onset in adolescents and early school start times are associated with a variety of negative consequences including reduced academic performance, anxiety, depression, social skills deficits, behavioral disturbances, and mood disturbances (Owens, 2008; Wolfson \& Carskadon, 1998). Research has begun to specify characteristics of the relationship between sleep disturbances and behavior problems in children (Aronen, Paavonen, Fjallberg, Soininen, \& Torronen, 2000; Owens, 2008). Specifically, children with sleep disturbances are more likely to struggle with aggression, conduct problems, inattention, and hyperactivity (Chervin, Dillon, Hedger Archbold, \& Ruzicka, 2003; Chervin et al., 2002). This is of particular relevance to mental health care providers, as behavior problems and disruptive behavior disorders are some of the most common reasons for referrals in pediatric mental health clinics (Verhulst \& Van Der Ende, 1997; Wu et al., 1999).

Although many children and adolescents experience sleep problems, they are more prevalent among children and adolescents with almost all psychiatric conditions, and they may increase the severity of the psychiatric conditions (Owens, 2008). Sleep problems are also symptoms of several disorders including depression, anxiety, and post-traumatic stress disorder (American Psychiatric Association, 2013). Ivanenko, Crabtree, O’Brien, \& Gozal (2006) examined the correlation between sleep problems in children with and without formal psychiatric diagnoses and found that children with known psychiatric disorders had more sleep disturbances compared to the non-psychiatric control group. Specifically, they found that children with a diagnosis of ADHD and comorbid anxiety and 
mood disorders as well as those with anxiety and mood disorders alone had higher rates of nocturnal awakenings and nightmares (Ivanenko, Crabtree, O'Brien, \& Gozal, 2006). In a meta-analysis of sleep in children with ADHD, Cortese, Faraone, Konofal, \& Lecendreux (2009) found that children with ADHD had significantly more sleep disturbances than controls with regards to bedtime resistance, sleep onset delay, night awakenings, morning awakenings, sleep disordered breathing, and daytime sleepiness. In a study by Alfano, Pina, Zerr, \& Villalta (2010), 85\% of parents of children with anxiety disorders reported significant sleep problems, and among them, children with Separation Anxiety Disorder and Generalized Anxiety Disorder experienced the highest amounts of sleep problems. Additionally, Lavigne et al. (1999) found a relationship between lack of sleep in young children and a clinical diagnosis especially for Oppositional Defiant Disorder. Studies have also found that patients with Oppositional Defiant Disorder and Conduct Disorder had more sleep problems than their non-clinical peers (Aronen et al., 2000; Aronen, Lampenius, Fontell, \& Simola, 2014). The direction of causality between psychiatric disorders and sleep disturbances has been debated. However, the importance of treating sleep problems to improve well-being is well established, as treating sleep disturbances can directly impact and improve an individual's functioning and mental health.

Although sleep disturbances have been documented in a variety of psychiatric disorders, there is limited research regarding sleep characteristics among children and adolescents who present to mental health agencies for a first appointment prior to receiving a clinical diagnosis. Many sleep problems past infancy often are not discussed by parents with children's pediatricians, which leads to many sleep disturbances going undetected and untreated (Waters, Suresh, \& Nixon, 2013; Mindell \& Owens, 2003). It has also been well documented that many sleep problems in infants and young children tend to persist over time without treatment, highlighting the importance of timely treatment (Lam, Hiscock, \& Wake, 2003; Mindell et al., 2006; Owens, 2008). In a 4 year follow-up study of Finnish children, Simola, Liukkonen, Pitkaranta, Pirinen, \& Aronen (2012) found that children with sleep problems that continued from preschool to the school aged years had a 16-fold increase risk in attention problems, social problems, aggression, somatic complaints, and anxious/depressed moods. Thus, appropriate, early screening and assessment of sleep and identification of possible sleep disturbances in treatment settings is vital (Waters et al., 2013).

Improved understanding of possible contributing factors such as other presenting problems and use of electronics and television that may impact sleep is also needed. Many children and adolescents spend significant time watching television and/or playing videogames in their bedrooms, and some studies have found these activities to correlate with sleep disturbances (Cain \& Gradisar, 2010; Van den Bulck, 2004). For example, Owens et al. (1999) found that increased television watching at bedtime and having a television in one's bedroom were associated with bedtime resistance, sleep onset delay, anxiety about sleep, and reduced sleep duration. Furthermore, $90 \%$ of the studies in a recent litera- 
ture review found that screen time is adversely associated with sleep outcomes; most notably shortened sleep duration and delayed timing (Hale \& Guan, 2015).

Despite the vital importance of sleep, many mental health agency intake and diagnostic assessment protocols lack comprehensive assessments of sleep characteristics leading to missed opportunities to address specific sleep problems in this population. In fact, research has found that many health professionals, including pediatricians and psychologists who work with children, do not receive adequate training regarding pediatric sleep and are not well equipped for assessing and/or treating sleep issues in this population (Schreck \& Richdale, 2011; Owens, 2001; Mindell \& Owens, 2003; Meltzer, Phillips, \& Mindell, 2009). Understanding more about the sleep characteristics of children and adolescents utilizing mental health services is critical, as the impact of sleep and sleep disturbance on functioning and well-being is significant. For both pediatric health and mental health care providers, obtaining information about sleep is valuable in helping children, adolescents, and their parents identify specific sleep disturbances which can lead to a more accurate diagnosis, as well as identification of sleep related treatment goals.

The purpose of the study was to better understand characteristics of sleep and their relationships to media use and other presenting emotional and behavior problems in children and adolescents seeking mental health services. In light of past research demonstrating that sleep disturbances in youth often go undetected and untreated, as well as previous findings that sleep issues are more prevalent among youth with mental health issues, it was hypothesized that the study sample would also be experiencing significant sleep disturbances in addition to the mental health concerns that brought them to the clinic. The study further aims to gather information about prevalence and qualities of sleep, use of electronics at bedtime, and comorbid problems that may be addressed in mental health assessments and interventions.

\section{Materials and Methods}

Table 1 presents demographic characteristics for the total sample. Participants were primary caregivers of children and adolescents aged 2 - 18 years seeking outpatient mental health services at a mental health clinic for children, adolescents and families located within a university medical center in the Midwestern United States. The clinic is located in a free-standing building on a university medical campus. The clinic is dedicated to child and adolescent mental health treatment, with separate inpatient, partial hospitalization, and outpatient programs. A range of professionals work in the outpatient division, including licensed social workers, psychologists, and counselors, as well as board certified child and adolescent psychiatrists. In addition, trainees including child and adolescent psychiatry residents, counseling interns, social work interns, and psychology externs see patients under supervision in the clinic. Services provided include outpatient evaluations, individual and family therapy, medication 
Table 1. Participant ethnicity.

\begin{tabular}{cc}
\hline & Valid Percent \\
\hline American Indian & 0.8 \\
Hispanic & 2.8 \\
White & 70.8 \\
Black & 10.8 \\
Other & 0.8 \\
Biracial & 14.0 \\
\hline
\end{tabular}

management, psychological assessment, group therapy, and case management. Although the clinic is located in an urban setting, it regularly serves families from a radius of approximately 50 - 60 miles from rural, suburban, and urban contexts. Patients range in age from infancy through early adulthood. The clinic typically serves $70 \%$ patients enrolled in Medicaid, 29\% with third party insurance, and $1 \%$ uninsured.

The study measure included demographic questions regarding age, gender, and ethnicity. In addition, there were four questions about sleep, including, "On average, about how many hours of sleep does your child get per night" ("sleep duration"), "How long does it take your child to fall asleep once in bed" ("sleep latency," reported in minutes), "On average, how many times does your child wake up during the night" ("night wakings"), and "How many nightmares does your child have in an average week?" Caregivers also were asked to report on the amount of television their child views on weekdays and weekends, videogame, computer, or hand held electronic game use time amounts on weekdays and weekends, and whether the child has access to each type of device in their bedrooms. Finally, caregivers were asked whether their child currently experiences the following problems: defiant and oppositional behavior, temper tantrums, aggression, behavior problems in school, sadness, irritability, worries/fears, mood swings, developmental delays, poor school performance, hyperactivity, inattention/concentration problems, parental divorce/family conflict, social/peer problems, and other problems.

The study measure was administered at outpatient intake appointments. The primary caregivers of 1772 children and adolescents were asked to voluntarily fill out the study measure, and 256 of those caregivers completed the measure, while 1516 refused or failed to complete and return the measure. To protect their anonymity, caregivers were asked not to write their names or other personally identifying information on the study measure. They were told the completion of the measure was voluntary and they may choose to return it blank. They were asked to complete the measure only with reference to the children and adolescents who were presenting for outpatient services on that date. When the measure was complete, they returned the form into a covered box located at the desk of the outpatient secretary. Caregivers were not compensated for their participation. Because the study measure was completed by caregivers voluntarily and 
anonymously, informed consent from participants was not obtained. Furthermore, there was no way to follow-up with parents on why they did not return the measure or whether type of insurance or other socioeconomic variables impacted completion rates. The study was reviewed and designated as exempt by the principle investigator's Investigational Review Board (IRB). There were no exclusion criteria for the study.

\section{Results}

Independent samples t-tests were conducted in order to examine gender differences for the sleep variables. Males and female participants did not differ significantly on the variables sleep duration (hours of sleep per night), sleep latency (average number of minutes before sleep onset), or nightmares. However, female patients were reported to have more frequent night wakings (average number of wakings per night) ( $M=1.31 \mathrm{vs.} 0.90 ; t=2.59 ; p=.01)$ than were male patients. Bivariate correlations indicated that sleep duration is negatively correlated with age $(r=-.24 ; p<.0001)$, but other sleep variables were not related to age.

For descriptive purposes and because sleep requirements vary by age group, the sample was divided into the following age groups: 2 - 4 years, 5 - 12 years, and 13 - 18 years. Table 2 lists the characteristics of sleep by age group. Analysis of variance indicated that sleep duration differed significantly by age group ( $F=$ 7.33; $d f=3245, p<.0001)$. No statistically significant differences were found on the other sleep variables. Results showed sleep deficits across all three age groups when compared to established sleep requirements for each age group. For instance, $6 \%$ of the sample had an average of 4 or less hours per night, while $16 \%$ of the sample had less than 6 or less hours per night. Table 3 depicts mean sleep

Table 2. Sleep characteristics by age.

\begin{tabular}{|c|c|c|c|}
\hline & $\begin{array}{c}2-4 \text { years } \\
(\mathrm{n}=17)\end{array}$ & $\begin{array}{c}5-12 \text { years } \\
(n=149)\end{array}$ & $\begin{array}{c}13-18 \text { years } \\
(\mathrm{n}=86)\end{array}$ \\
\hline & $M(S D)$ & $M(S D)$ & $M(S D)$ \\
\hline Sleep Duration (hours) & $8.32(1.32)$ & $8.14(1.68)$ & $7.28(1.54)$ \\
\hline Sleep Latency (minutes) & $63.68(57.32)$ & $50.49(46.40)$ & $57.54(74.13)$ \\
\hline $\begin{array}{c}\text { Night Wakings } \\
\text { (number per night) }\end{array}$ & $.68(.90)$ & $1.02(1.81)$ & $1.13(1.20)$ \\
\hline Nightmares (number per week) & $0.62(0.77)$ & $0.73(2.00)$ & $1.94(5.66)$ \\
\hline
\end{tabular}

Note. 4 subjects did not identify age.

Table 3. Sleep duration across age groups.

\begin{tabular}{cccc}
\hline $\begin{array}{c}\text { Age group } \\
\text { (years) }\end{array}$ & $\begin{array}{c}\text { Suggested Hours of } \\
\text { Sleep* }\end{array}$ & Average $(S D)$ & $\begin{array}{c}\text { Percentage of Children Below the } \\
\text { Recommended Hours of Sleep (\%) }\end{array}$ \\
\hline $2-4$ & $11-12$ & $8.32(1.32)$ & 94 \\
$5-12$ & 10 & $8.14(1.68)$ & 83 \\
$13-18$ & $9-10$ & $7.28(1.54)$ & 100 \\
\hline
\end{tabular}

Note. ${ }^{\star}$ Based on National Heart, Lung, and Blood Institute (2017). 
durations for the age groups compared to the recommended sleep amounts per age, as well as the percentage of youths in the sample who had sleep durations below the recommended hours of sleep.

Regarding mental health problems endorsed, Table 4 lists the percent of the total sample that reported each problem. The problems listed by caregivers were added to determine the total number of problems endorsed for each patient. The average number of problems endorsed by caregivers was $5.7(N=256 ; x=5.73$; $S D=3.01)$. Bivariate correlations were then conducted to examine whether significant associations exist between total number of problems listed and sleep duration for each age group. In the youngest ( 2 - 4 years) age group, sleep duration was negatively associated with the total number of problems listed $(r=-.49$; $p=.047)$. Similarly, for school-aged children (5 - 12 years), sleep duration was negatively associated with number of problems listed $(r=-0.22 ; p=.008)$. In contrast, adolescents' sleep duration was not correlated with number of problems reported.

Independent samples t-tests were conducted in order to compare groups of participants with and without presenting problems on each of the sleep variables. Participants with worries and fears had significantly lower sleep duration $(M=7.61$ vs. 8.09; $t=-2.29, p=.023)$, and those with irritability had significantly lower sleep duration than those without ( $M=7.67$. vs. 7.68; $t=-2.24, p$ $=.026)$. Children and adolescents with mood swings also reported significantly less sleep than those without ( $M=7.61 v s .8 .21 ; t=-2.90, p=.004)$. Groups with and without sadness did not differ on sleep duration, but there were significant

Table 4. Percent of the total sample with caregiver-reported problems.

\begin{tabular}{cc}
\hline Problem & Percent \\
\hline Defiant/Oppositional & 48.0 \\
Tantrums & 34.8 \\
Aggression & 41.0 \\
Behavioral Problems in School & 34.4 \\
Sadness & 44.1 \\
Irritability & 57.8 \\
Worries/Fears & 45.3 \\
Mood Swings & 57.0 \\
Developmental Delays & 16.4 \\
Poor School Performance & 35.0 \\
Hyperactivity & 39.1 \\
Inattention & 60.9 \\
Parental Divorce/Family Conflict & 23.0 \\
Social/Peer Problems & 30.9 \\
Other Problems & 5.1 \\
\hline
\end{tabular}


differences in sleep latency indicating that participants with sadness take more time to fall asleep than those without sadness ( $M=67.83$ vs. $M=45.55 ; t=2.83$, $p=.005)$.

Independent samples t-tests were also conducted in order to compare groups of participants with and without electronic devices and televisions in their bedrooms. Results indicate that participants with televisions in the bedroom reported significantly less hours of sleep than those without a television in the bedroom $(M=7.71$ vs. $8.13 t=-1.99, p=.047)$. Bivariate correlations were conducted to determine if relationships exist between average hours of device use and sleep variables. The average number of hours of television watched per week was positively correlated with the number of minutes of sleep latency ( $r$ $=.20, p=.003)$. Time spent playing videogames was negatively correlated with sleep duration $(r=-.200, p=.003)$.

\section{Discussion}

This study explored various characteristics of sleep and other presenting problems in children and adolescents seeking mental health services within an outpatient clinic. Due to previous findings in the literature that sleep problems in children and adolescents often go undetected and that sleep disturbances are more prevalent in youth experiencing a host of mental health issues, it was hypothesized that this study sample would also be experiencing sleep disturbances as well as the mental health issues that prompted their visit to the clinic. Factors including use of electronics among children and adolescents before bedtime were also explored due to previous research finding a link between electronics usage and sleep characteristics. Consistent with previous research, the study results indicated that sleep disturbances are significant issues impacting youth who present for mental health services. Specifically, caregivers of children and adolescents endorsed many sleep problems including reduced sleep duration, lengthy sleep onset delay, night wakings, and nightmares. One of the most important findings was the high percentage of the sample that had inadequate sleep duration relative to recommended guidelines. For instance, $16 \%$ of the population surveyed had less than 6 hours per night. This is significantly less than the recommended sleep guidelines for each age group, indicating that a substantial percentage of this sample was sleep deprived. The study found that sleep duration was negatively correlated with age, which is consistent with previous research indicating that children require less sleep as they age. However, the study found that the duration of total sleep obtained for all three age groups surveyed was significantly lower than expected compared to sleep requirements by age even when considering natural declines in sleep needs. There were no significant differences between males and females related to sleep duration.

Another important finding regarding the total sleep duration for children between the ages of 2 - 4 was identified. While toddlers and preschoolers in this age group should be getting approximately $11-12$ hours of sleep per night, 
children in this study reportedly received an average of 8.32 hours per night, indicating an approximately 3 to 4 hour deficit on average. Furthermore, children between the ages of $5-12$ received an average of 8.14 hours per sleep per night, which is also significantly less than the 10 hours of sleep recommended for their age (National Heart, Lung, and Blood Institute, 2017). Adolescents averaged 7.28 hours of sleep per night, which is also less than the 9 - 10 hours per day recommended for their age. This has significant implications for the health and wellbeing of children as research has identified many detrimental consequences to one's physical and mental health to be associated with short sleep duration.

Results showed an average sleep latency of almost one hour per night. In the literature, sleep onset delay is usually defined as taking 30 minutes or more to fall asleep (Van der Heijden, Smits, Van Someren, Ridderinkhof, \& Gunning, 2007). Thus, children and adolescents in this study, on average, have substantial sleep latencies, which likely contributes to the reduced total sleep duration. Children in the youngest age range $(2-4)$ had the longest sleep latency of 63.68 minutes per night. Regarding night wakings, the average number per night across all 3 age groups was 1 . The average number of nightmares per week was also 1 across all 3 age groups. Although no gender differences were seen with nightmares, females were found to have a higher number of wakings per night than males. Both increased nightmares and wakings are also problematic as they impact and reduce overall sleep duration and may contribute to daytime fatigue and distress.

The study also examined the types and numbers of problems that children and adolescents were experiencing at the time of their first appointment. The average number of problems endorsed per patient was 5.7, indicating that the study sample was experiencing a variety of presenting problems in addition to sleep difficulties. When examining the correlations between presenting problems, age group, and sleep characteristics, many interesting findings were revealed. For instance, hours of sleep duration were negatively associated with the total number of problems endorsed for children in the $2-4$ and $5-12$ groups. Although direction of causation could not be determined, it is possible that sleep deprivation may be a major contributing factor to the amount of mental health issues and problems that younger children are experiencing. Alternatively, it is possible the mental health problems cause sleep problems. Furthermore, it was found that participants with worries and fears, irritability, and mood swings had lower amounts of sleep than youths without those concerns. This is consistent with the diagnostic criteria for both anxiety and mood disorders that highlight potential sleep disturbances as part of both disorders. Consequently, individuals who present with these concerns especially would benefit from a thorough assessment of their sleep. Although individuals who experience sadness did not differ on sleep duration, they did experience higher sleep latencies. Thus, sadness may be a major contributing factor in sleep latency, or sadness may in part result from difficulty falling asleep. Although the direction of causality cannot be 
established, these findings highlight the important relationship between untreated mental health issues and sleep disturbances.

As previously mentioned, electronics and media usage in bedrooms are considered to be contributing factors to sleep disturbances in the general population. Results of this study were consistent with past research and indicated that sleep duration was lower for children and youths with televisions in their bedrooms. Additionally, the more hours that youth spent watching television, the longer it took to fall asleep. Amount of time spent playing videogames also was related to lower duration of sleep in youths. These results highlight the importance of assessment of media use for youths seeking mental health services.

\section{Limitations}

Several limitations of this study should be noted. First, the low participation rate may have impacted the results and limited the study's generalizability. The study measure was developed for this study, and did not have established reliability and validity. The study measure also relied only on caregiver report which was subjective, and caregivers may not have been fully aware of sleep problems or electronic use at bedtime. For instance, caregivers may not have had an accurate understanding about their child's night wakings or sleep latency that typically occur when the caregiver is not present. Additionally, the study measure used retrospective accounts of children and adolescents' sleep and problems, which may have influenced the accuracy of the results. More objective measures of sleep would also increase accuracy. Finally, the study measure should have specified cell phone usage instead of the general category of hand held devices as many children and adolescents have their own phones and may be using them instead of other hand held electronics. With the increased usage of texting and social media, future studies should specifically examine the impact that cell phone use at night has on sleep and overall functioning. Because implications about causality were not possible with this design, it is recommended that future research employ a longitudinal design that can better track the timing of changes in sleep and mental health problems.

Future research should include the addition of a well-established, more objective sleep measure for parents to complete, as well as for older children and adolescents to complete on their own sleep patterns. More comprehensive tracking of media/electronic usage including comparing phone vs. other electronic devices would also be advised. Future research should also employ follow-up measures to examine changes and improvements after treatment is initiated.

\section{Conclusion}

The results of this study indicate that in general, children and adolescents who are brought by their caregivers for outpatient mental health treatment are likely to be experiencing many sleep disturbances in addition to the other concerns that led to their first appointment. Overall, this sample appeared to have signifi- 
cant sleep deficits due to reduced sleep duration, increased sleep latency, night wakings, and nightmares. Patients also experienced numerous problems as endorsed on the study measure with inattention, irritability, mood swings, and defiant and oppositional problems being some of the most prevalent problems reported. The comorbid issues identified indicate that many youth have multiple problems that need to be formally assessed and addressed in treatment. Preschool and school aged children experienced the greatest sleep deficits and experienced higher numbers of problems. Findings also indicated that usage of television and electronics use may be contributing to sleep disturbances, suggesting that questions about media use should be included in the assessment as well. This is of particular importance because many parents are likely to be unaware of the detrimental effects that media use and electronics may have on their child's sleep.

Previous literature has reported the multitude of negative consequences that result from sleep disturbances in children, as well as the fact that sleep disturbances frequently go undetected in the primary care setting. Based on this study's findings, it is imperative that clinicians in mental health specifically perform a comprehensive sleep assessment and not just inquire about sleep disturbances as part of the diagnostic criteria for certain disorders. Sleep hygiene and possible sleep disturbances need to be fully explored as they may be significant contributing factors to overall emotional and behavioral health of young patients. In-depth questions about sleep should be incorporated within outpatient mental health/psychiatric evaluation intake forms and in later follow-up appointments. Media and electronics usage at night time should also be inquired about due to their potential negative impact on sleep. Furthermore, sleep disturbances should be specifically targeted in treatment plans aside from the more overt problems parents endorse and are seeking services for. This will also serve as a tool to educate caregivers on appropriate sleep requirements and to help improve their child's sleep hygiene.

\section{Acknowledgements}

We would like to thank Karyssa Schrouder and Pooja Sheth for contributing to this paper.

\section{References}

Alfano, C. A., Pina, A. A., Zerr, A. A., \& Villalta, I. K. (2010). Pre-Sleep Arousal and Sleep Problems of Anxiety-Disordered Youth. Child Psychiatry Human Development, 41, 156-167. https://doi.org/10.1007/s10578-009-0158-5

American Psychiatric Association (2013). Diagnostic and Statistical Manual of Mental Disorders (5th ed.). Arlington, VA: American Psychiatric Publishing. https://doi.org/10.1176/appi.books.9780890425596

Aronen, E. T., Lampenius, T., Fontell, T., \& Simola, P. (2014). Sleep in Children with Disruptive Behavior Disorders. Behavioral Sleep Medicine, 12, 373-388.

https://doi.org/10.1080/15402002.2013.821653 
Aronen, E. T., Paavonen, E. J., Fjallberg, M., Soininen, M., \& Torronen, J. (2000). Sleep and Psychiatric Symptoms in School-Age Children. Journal of the American Academy of Child and Adolescent Psychiatry, 39, 502-508. https://doi.org/10.1097/00004583-200004000-00020

Cain, N., \& Gradisar, M. (2010). Electronic Media Use and Sleep in School-Aged Children and Adolescents: A Review. Sleep Medicine, 11, 735-742. https://doi.org/10.1016/j.sleep.2010.02.006

Chervin, R. D., Dillon, J. E., Hedger Archbold, K., \& Ruzicka, D. L. (2003). Conduct Problems and Symptoms of Sleep Disorders. Children Journal of the American Academy of Child \& Adolescent Psychiatry, 42, 201-208. https://doi.org/10.1097/00004583-200302000-00014

Chervin, R. D., Hedger Archbold, K., Dillon, J. E., Panahi, P., Pituch, K. J., Dahl, R. E., \& Guilleminault, C. (2002). Inattention, Hyperactivity and Symptoms of Sleep Disorder Breathing. Pediatrics, 109, 449-456. https://doi.org/10.1542/peds.109.3.449

Cortese, S., Faraone, S.V., Konofal, E., \& Lecendreux, M. (2009). Sleep in Children with Attention-Deficit Hyperactivity Disorder: Meta-Analysis of Subjective and Objective Studies. Journal of the American Academy of Child and Adolescent Psychiatry, 48, 894-908. https://doi.org/10.1097/CHI.0b013e3181ae09c9

Fallone, G., Owens, J. A., \& Deane, J. (2002). Sleepiness in Children and Adolescents: Clinical Implications. Sleep Medicine Reviews, 6, 287-306. https://doi.org/10.1053/smrv.2001.0192

Hale, L., \& Guan, S. (2015). Screen Time and Sleep among School-Aged Children and Adolescents: A Systematic Literature Review. Sleep Medicine Reviews, 21, 50-58. https://doi.org/10.1016/j.smrv.2014.07.007

Ivanenko, A., Crabtree, V. M., O’Brien, L. M., \& Gozal, D. (2006). Sleep Complaints and Psychiatric Symptoms in Children Evaluated at a Pediatric Mental Health Clinic. Journal of Clinical Sleep Medicine, 2, $42-48$.

Lam, P., Hiscock, H., \& Wake, M. (2003). Outcomes of Infant Sleep Problems: A Longitudinal Study of Sleep, Behavior, and Maternal Well-Being. Pediatrics, 111, e203-e207. https://doi.org/10.1542/peds.111.3.e203

Lavigne, J. V., Arend, R., Rosenbaum, D., Smith, A., Weissbluth, M., Binns, H. J., \& Christoffel, K. K. (1999). Sleep and Behavior Problems among Preschoolers. Journal of Developmental and Behavioral Pediatrics, 20, 164-169. https://doi.org/10.1097/00004703-199906000-00005

Meltzer, L. J., Phillips, C., \& Mindell, J. A. (2009). Clinical Psychology Training in Sleep and Sleep Disorders. Journal of Clinical Psychology, 65, 305-318. https://doi.org/10.1002/jclp.20545

Mindell, J. A., \& Owens, J. A. (2003). Sleep Problems in Pediatric Practice: Clinical Issues for the Pediatric Nurse Practitioner. Journal of Pediatric Health Care, 17, 324-331. https://doi.org/10.1016/j.pedhc.2003.09.003

Mindell, J. A., Kuhn, B., Lewin, D., Meltzer, L., \& Sadeh, A. (2006). Behavioral Treatment of Bedtime Problems and Night Wakings in Infants and Young Children. Sleep, 29, 1263-1276.

National Heart, Lung, and Blood Institute (2017). How Much Sleep Is Enough? http://www.nhlbi.nih.gov/health/health-topics/topics/sdd/howmuch.html

Owens, J. (2001). The Practice of Pediatric Sleep Medicine: Results of a Community Survey. Pediatrics, 108, e51-e51. https://doi.org/10.1542/peds.108.3.e51

Owens, J. (2008). Classification and Epidemiology of Childhood Sleep Disorders. Primary 
care: Clinics in Office Practice, 35, 533-546. https://doi.org/10.1016/j.pop.2008.06.003

Owens, J., Maxim, R., McGuinn, M., Nobile, B. A., Msall, M., \& Alario, A. (1999). Television-Viewing Habits and Sleep Disturbances in School Children. Pediatrics, 104, e27. http://pediatrics.aappublications.org/content/104/3/e27 https://doi.org/10.1542/peds.104.3.e27

Schreck, K. A., \& Richdale, A. L. (2011). Knowledge of Childhood Sleep: A Possible Variable in under or Misdiagnosis of Childhood Sleep Problems. Journal of Sleep Research, 20, 589-597. https://doi.org/10.1111/j.1365-2869.2011.00922.x

Simola, P., Liukkonen, K., Pitkaranta, A., Pirinen, T., \& Aronen, E. T. (2012). Psychosocial and Somatic Outcomes of Sleep Problems in Children: A 4-Year Follow-Up Study. Child: Care, Health and Development, 40, 60-67. https://doi.org/10.1111/j.1365-2214.2012.01412.x

Van den Bulck, J. (2004). Television Viewing, Computer Game Playing, and Internet Use and Self-Reported Time to Bed and Time out of Bed in Secondary-School Children. Sleep, 27, 101-104. https://doi.org/10.1093/sleep/27.1.101

Van der Heijden, K. B., Smits, M. G., Van Someren, E. J., Ridderinkhof, K. R., \& Gunning, W. B. (2007). Effects of Melatonin on Sleep, Behavior, and Cognition in ADHD and Chronic Sleep-Onset Insomnia. Journal of the American Academy of Child \& Adolescent Psychiatry, 46, 233-241. https://doi.org/10.1097/01.chi.0000246055.76167.0d

Verhulst, F. C. \& Van Der Ende, J. (1997). Factors Associated with Child Mental Health Service Use in the Community. Journal of the American Academy of Child and Adolescent Psychiatry, 36, 901-909. https://doi.org/10.1097/00004583-199707000-00011

Waters, K. A., Suresh, S., \& Nixon, G. M. (2013). Sleep Disorders in Children. Medical Journal of Australia, 199, S31-S35. https://doi.org/10.5694/mja13.10621

Wolfson, A. R., \& Carskadon, M. A. (1998). Sleep Schedules and Daytime Functioning in Adolescents. Child Development, 69, 875-887. https://doi.org/10.1111/j.1467-8624.1998.tb06149.x

Wu, P., Hoven, C. W., Bird, H. R., Moore, R. E., Cohen, P., Alegria, M., Roper, M. T. et al. (1999). Depressive and Disruptive Disorders and Mental Health Service Utilization in Children and Adolescents. Journal of the American Academy of Child and Adolescent Psychiatry, 38, 1081-1090. https://doi.org/10.1097/00004583-199909000-00010 